\title{
SOME LIMIT THEOREMS FOR GENERALIZED ALLOCATION SCHEMES
}

\author{
ISTVÁN FAZEKAS AND BETTINA PORVÁZSNYIK
}

Received 11 December, 2014

\begin{abstract}
In this paper two modifications of Kolchin's generalized allocation scheme are studied. Results known for Kolchin's scheme are extended to the new models. Representation theorems, strong laws of large numbers and local limit theorems are obtained. In the proofs some general inequalities are used.
\end{abstract}

2010 Mathematics Subject Classification: 60C05; 60F05

Keywords: Generalized allocation scheme, law of large numbers, normal limit theorem

\section{INTRODUCTION}

The generalized allocation scheme was introduced by V. F. Kolchin. Its properties and applications are described in the monographies [12] and [14]. The usual allocation scheme (see $[1,2,13]$ ), the random forests and other random structures are special cases of Kolchin's scheme (see [12] and [14]). The scheme itself is defined as follows (we call it Model 1).

Model 1

Let $\eta_{1}, \eta_{2}, \ldots, \eta_{N}$ be nonnegative integer-valued random variables. If there exist independent identically distributed random variables $\xi_{1}, \xi_{2}, \ldots, \xi_{N}$ such that the joint distribution of $\eta_{1}, \eta_{2}, \ldots, \eta_{N}$ admits the representation

$$
\begin{gathered}
\mathbb{P}\left\{\eta_{1}=k_{1}, \ldots, \eta_{N}=k_{N}\right\}= \\
=\mathbb{P}\left\{\xi_{1}=k_{1}, \ldots, \xi_{N}=k_{N} \mid \sum_{i=1}^{N} \xi_{i}=n\right\},
\end{gathered}
$$

where $k_{1}, k_{2}, \ldots, k_{N}$ are arbitrary non-negative integers with $\sum_{i=1}^{N} k_{i}=n$, we say that the distribution of $\eta_{1}, \eta_{2}, \ldots, \eta_{N}$ is represented by the generalized allocation scheme with parameters $n$ and $N$, and independent random variables $\xi_{1}, \xi_{2}, \ldots, \xi_{N}$.

The first author was supported by the TÁMOP-4.2.2.C-11/1/KONV-2012-0001 project. The project has been supported by the European Union, co-financed by the European Social Fund.

The second author was supported by the European Union and the State of Hungary, co-financed by the European Social Fund in the framework of TÁMOP 4.2.4.A/2-11-1-2012-0001 "National Excellence Program'. 
This scheme can be considered as the allocation of $n$ balls into $N$ boxes. The random variable $\eta_{i}$ can be interpreted as the number of balls in the $i$ th box. The detailed description of the properties of scheme (1.1) are given in [12] and [14]. The importance of scheme (1.1) is its connection with random graphs. Further properties of scheme (1.1) can be found in the papers of V. F. Kolchin and A. V. Kolchin (see e.g. [10,11]). In [5] a strong law of large numbers (SLLN) was proved for Model 1. Using that SLLN one can obtain SLLN-s for usual random allocations and random forests. We mention that the original proofs of SLLN-s for usual allocations and random forests needed very long elementary calculations (see [2] and [3]).

In [7] the following analogue of Kolchin's scheme was studied.

Model 2

Consider random variables $\eta_{1}, \eta_{2}, \ldots, \eta_{N}$ with joint distribution

$$
\begin{gathered}
\mathbb{P}\left\{\eta_{1}=k_{1}, \ldots, \eta_{N}=k_{N}\right\}= \\
=\mathbb{P}\left\{\xi_{1}=k_{1}, \ldots, \xi_{N}=k_{N} \mid \sum_{i=1}^{N} \xi_{i} \leq n\right\},
\end{gathered}
$$

where $k_{1}, \ldots, k_{N}$ are arbitrary non-negative integers with $\sum_{i=1}^{N} k_{i} \leq n$. In this case, we place at most $n$ balls into $N$ boxes. In [7] strong laws of large numbers, normal and Poisson limit theorems for the number of boxes containing a fixed number of balls were obtained. The distribution of the maximum number of balls contained in the boxes was studied in [6].

In [8], another version of Kolchin's scheme was studied (see Model 3 below). In that modification, the condition in (1.1) was changed for $\sum_{i=1}^{N} \xi_{i} \geq n$. For Model 3 normal and Poisson local limit theorems were obtained in [8].

In this paper our aim is to study two modifications of Kolchin's model. We extend certain results of [7] and [6] for these models. In Section 2.1 we review Model 3 and we present some new results for it. Actually, we obtain strong laws (Theorems 1 and 2) and a local limit theorem (Theorem 3). In Section 2.2 we introduce a new version of Kolchin's scheme (Model 4). Model 4 is a very general scheme. It contains Model 1, Model 2 and Model 3 as special cases. We find representation theorems and some limit theorems for Model 4. Finally, in Section 3 we present our proofs and some auxiliary results. In forthcoming research we intend to apply Model 4 to describe certain properties of random graphs.

\section{NOTATIONS AND MAIN RESULTS}

We shall apply o(.) and $\mathrm{O}($. $)$ in the usual sense, that is $k_{n}=\mathrm{o}\left(l_{n}\right)$ if $\lim _{n \rightarrow \infty} k_{n} / l_{n}=$ 0 and $k_{n}=\mathrm{O}\left(l_{n}\right)$ if the sequence $k_{n} / l_{n}$ is bounded. 
Let us denote by $\mu_{n N}$ the number of cases when $\left\{\eta_{i}=r\right\}$, where $r$ is a fixed integer, $r \in\{0,1, \ldots, n\}$. Then

$$
\mu_{n N}=\sum_{i=1}^{N} \mathbb{I}_{\left\{\eta_{i}=r\right\}}
$$

can be interpreted as the number of boxes containing $r$ balls. Here $\mathbb{I}_{A}$ denotes the indicator of the set $A$.

Let $\xi_{0}$ be a random variable, $\mathbb{P}\left\{\xi_{0}=r\right\}=p_{r}$ and $\mathbb{E} \xi_{0}=a$. Let $\xi_{1}, \xi_{2}, \ldots, \xi_{N}$ be independent copies of $\xi_{0}$. Introduce notation $S_{N}=\sum_{i=1}^{N} \xi_{i}$ and $S_{N}^{c}=\sum_{i=1}^{N}\left(\xi_{i}-\right.$ a).

Denote by $\xi_{0}^{(r)}$ a random variable with distribution

$$
\mathbb{P}\left\{\xi_{0}^{(r)}=k\right\}=\mathbb{P}\left\{\xi_{0}=k \mid \xi_{0} \neq r\right\} .
$$

Let $\mathbb{E} \xi_{0}^{(r)}=a_{r}$ and let $\xi_{1}^{(r)}, \ldots, \xi_{N}^{(r)}$ be independent copies of $\xi_{0}^{(r)}$. Let $S_{N}^{(r)}=$ $\sum_{i=1}^{N} \xi_{i}^{(r)}$.

We see that the connection between the expectations $a$ and $a_{r}$ is $a_{r}=\frac{a-r p_{r}}{1-p_{r}}$.

\subsection{Model 3}

In this section we consider Model 3 that is we assume that (2.1) is satisfied.

Let $\eta_{1}, \eta_{2}, \ldots, \eta_{N}$ be random variables with joint distribution

$$
\begin{gathered}
\mathbb{P}\left\{\eta_{1}=k_{1}, \ldots, \eta_{N}=k_{N}\right\}= \\
=\mathbb{P}\left\{\xi_{1}=k_{1}, \ldots, \xi_{N}=k_{N} \mid \sum_{i=1}^{N} \xi_{i} \geq n\right\},
\end{gathered}
$$

where $k_{1}, \ldots, k_{N}$ are arbitrary non-negative integers with $\sum_{i=1}^{N} k_{i} \geq n$. Then we say that $\eta_{1}, \eta_{2}, \ldots, \eta_{N}$ obey the assumption of Model 3. In this case we place at least $n$ balls into $N$ boxes.

Model 3 was introduced in [8]. We shall see that appropriate versions of certain results of [7] are true for Model 3.

First we present SLLN-s for Model 3.

The following theorem is a version of Theorem 1 of [7].

Theorem 1 (Model 3.). Let $\mathbb{E} \xi_{0}=a<\infty$. Suppose that there exists a sequence $B_{N}, N=1,2, \ldots$, such that $B_{N} \rightarrow \infty, N \geq B_{N}>0$ for all $N=1,2, \ldots$,

$$
\lim _{N \rightarrow \infty} \frac{B_{N+1}}{B_{N}}=1
$$

and

$$
\frac{S_{N}^{c}}{B_{N}} \rightarrow \xi^{\prime} \text { as } N \rightarrow \infty
$$


in distribution, where $\xi^{\prime}$ is a random variable with distribution function $F$. Let $C$ be a point of continuity of $F$ such that $F(C)=\mathbb{P}\left\{\xi^{\prime}<C\right\}=q_{1}<1$. Then we have

$$
\lim _{n, N \rightarrow \infty, 0<\frac{n}{N}<C \frac{B_{N}}{N}+a} \frac{1}{N} \mu_{n N}=p_{r} \quad \text { almost surely. }
$$

Corollary 1. (Model 3.) Let $\mathbb{E} \xi_{0}=a<\infty$. Let $C<0$. Then we have

$$
\lim _{n, N \rightarrow \infty, 0<\frac{n}{N}<a+C} \frac{1}{N} \mu_{n N}=p_{r} \quad \text { almost surely. }
$$

Corollary 2. (Model 3.) Suppose that $\mathbb{E} \xi_{0}^{2}<\infty, \mathbb{E} \xi_{0}=a$. Let $C \in \mathbb{R}$. Then we have

$$
\lim _{n, N \rightarrow \infty, 0<\frac{n}{N}<\frac{C}{\sqrt{N}}+a} \frac{1}{N} \mu_{n N}=p_{r} \quad \text { almost surely. }
$$

Recall that a random variable $\xi$ satisfies the Cramér's condition, if there exists a positive constant $H$ such that $\mathbb{E} e^{\lambda \xi}<\infty$ for all $|\lambda|<H$.

We have the following analogue of Theorem 2 of [7].

Theorem 2 (Model 3.). Let $\mathbb{E} \xi_{0}=a<\infty$.

(1) Let $\alpha<a$. Then

$$
\lim _{n, N \rightarrow \infty, \frac{n}{N} \rightarrow \alpha} \frac{1}{N} \mu_{n N}=p_{r} \quad \text { almost surely. }
$$

(2) Assume that $\xi_{0}$ satisfies the Cramér condition. Then

$$
\lim _{n, N \rightarrow \infty, \frac{n}{N} \rightarrow a} \frac{1}{N} \mu_{n N}=p_{r} \quad \text { almost surely. }
$$

Consider the random variable $\xi_{0}$ with the following power series distribution. Let $b_{0}, b_{1}, b_{2}, \ldots$ be a sequence of non-negative numbers and let $R$ denote the radius of convergence of the series

$$
B(\theta)=\sum_{k=0}^{\infty} \frac{b_{k} \theta^{k}}{k !} .
$$

Assume that $R>0$. We assume that $\xi_{0}=\xi_{0}(\theta)$ has distribution

$$
p_{k}=p_{k}(\theta)=\mathbb{P}\left\{\xi_{0}(\theta)=k\right\}=\frac{b_{k} \theta^{k}}{k ! B(\theta)}, k=0,1,2, \ldots
$$

We will assume that the distribution of the random variable $\xi_{0}(\theta)$ satisfies

$$
b_{0}>0, b_{1}>0 .
$$

(For more details, see e.g. [12].)

The following theorem is a version of Theorem 8 of [7]. 
Theorem 3 (Model 3.). Suppose that the random variable $\xi_{0}=\xi_{0}(\theta)$ has distribution (2.7) and condition (2.8) is satisfied. Suppose that $r=1$ and $n \geq 1$ is fixed. Let $N \rightarrow \infty$ such that $N p_{1}(\theta) \rightarrow \lambda$ for some $0<\lambda<\infty$. Then for all $k \in \mathbb{N}_{0}$ we have

$$
\mathbb{P}\left\{\mu_{n N}=k\right\}=\frac{\frac{\lambda^{k} e^{-\lambda}}{k !}}{\sum_{l=n}^{\infty} \frac{\lambda^{l} e^{-\lambda}}{l !}}(1+\mathrm{o}(1)) .
$$

\subsection{Model 4}

In this section we will study the following very general modification of Kolchin's scheme.

Consider random variables $\eta_{1}, \eta_{2}, \ldots, \eta_{N}$ with joint distribution

$$
\begin{gathered}
\mathbb{P}\left\{\eta_{1}=k_{1}, \ldots, \eta_{N}=k_{N}\right\}= \\
=\mathbb{P}\left\{\xi_{1}=k_{1}, \ldots, \xi_{N}=k_{N} \mid \xi_{1}+\cdots+\xi_{N} \in B_{n}\right\},
\end{gathered}
$$

where $k_{1}, \ldots, k_{N}$ are arbitrary non-negative integers with $\sum_{i=1}^{N} k_{i} \in B_{n}$ and $B_{n}$ is a nonempty subset of $\mathbb{R}_{+}$. Then we say that $\eta_{1}, \ldots, \eta_{N}$ obey a generalized allocation scheme of type Model 4.

Remark 1. Here we assume that $\mathbb{P}\left\{\sum_{i=1}^{n} \xi_{i} \in B_{n}\right\}>0$.

We have the following analogue of Kolchin's formula in Model 4 (see Lemma 1.2.1 of [12]).

Lemma 1 (Model 4.). For all $k=0,1,2, \ldots, N$ we have

$$
\mathbb{P}\left\{\mu_{n N}=k\right\}=\left(\begin{array}{l}
N \\
k
\end{array}\right) p_{r}^{k}\left(1-p_{r}\right)^{N-k} \frac{\mathbb{P}\left\{S_{N-k}^{(r)} \in B_{n}-k r\right\}}{\mathbb{P}\left\{S_{N} \in B_{n}\right\}} .
$$

Let us denote by $\eta_{(N)}$ the maximal number of balls contained by any of the boxes, that is $\eta_{(N)}=\max _{1 \leq i \leq N} \eta_{i}$.

Consider the random variable $\xi_{0}^{(\leq r)}$ with distribution

$$
\mathbb{P}\left\{\xi_{0}^{(\leq r)}=k\right\}=\mathbb{P}\left\{\xi_{0}=k \mid \xi_{0} \leq r\right\} .
$$

Let $\xi_{i}^{(\leq r)}, i=1, \ldots, N$, be independent copies of $\xi_{0}^{(\leq r)}$. Let $S_{N}^{(\leq r)}=\sum_{i=1}^{N} \xi_{i}^{(\leq r)}$ and $\mathbb{E} \xi_{0}^{(\leq r)}=a_{\leq r}$.

We have the following representation of the distribution of $\eta_{(N)}$. This lemma is a version of Theorem 1 of [6].

Lemma 2 (Model 4.). We have

$$
\mathbb{P}\left\{\eta_{(N)} \leq r\right\}=\left(1-P_{r}\right)^{N} \frac{\mathbb{P}\left\{S_{N}^{(\leq r)} \in B_{n}\right\}}{\mathbb{P}\left\{S_{N} \in B_{n}\right\}},
$$

for all $r \in \mathbb{N}$, where $P_{r}=\mathbb{P}\left\{\xi_{0}>r\right\}$. 
In the following theorem we shall consider the case when the set $B_{n}$ is of the following form: $B_{n}=f(n) B$, where $n \in \mathbb{N}, B \subset \mathbb{R}_{+}$and $f(n)$ is a real-valued function of $n$.

Remark 2. If $f(n)=n$, then we have the following examples for Model 4.

- If $B=\{1\}$, then $B_{n}=\{n\}$, so we obtain the original Kolchin's model (Model 1).

- If $B=[0,1]$, then $B_{n}=[0, n]$, so we get Model 2 .

- If $B=[1, \infty)$, then $B_{n}=[n, \infty)$, so we get Model 3 .

Let $B^{\circ}$ be the set of inner points of $B$. We have the following local limit theorem for $\mu_{n N}$. This theorem is an appropriate version of Theorem 4 of [7].

Theorem 4 (Model 4 with $B_{n}=f(n) B$.). Let $0<\frac{N}{f(n)}<\infty$, for $n, N \in \mathbb{N}$. Let $\frac{N}{f(n)} \rightarrow \widetilde{\alpha_{1}}$ as $n, N \rightarrow \infty$, where $\widetilde{\alpha_{1}} \in \mathbb{R}_{+}$. Assume that $\widetilde{\alpha_{1}} a \in B^{\circ}$. Let $s_{r}^{2}=$ $p_{r}\left(1-p_{r}\right)$. Then

$$
\mathbb{P}\left\{\mu_{n N}=k\right\}=\frac{1}{\sqrt{2 \pi N} s_{r}} e^{-u^{2} / 2}(1+\mathrm{o}(1))
$$

as $n, N \rightarrow \infty$ so that $u=\frac{k-N p_{r}}{s_{r} N^{1 / 2}}$ belongs to an arbitrary bounded fixed interval.

Using Lemma 2, we can prove the following result. This theorem is an appropriate version of Theorem 2 of [6].

Theorem 5 (Model 4 with $B_{n}=f(n) B$.). Let $0<\frac{N}{f(n)}<\infty$ for $n, N \in \mathbb{N}$. Let $\frac{N}{f(n)} \rightarrow \widetilde{\alpha_{1}}$ as $n, N \rightarrow \infty$, where $\widetilde{\alpha_{1}} \in \mathbb{R}_{+}$. Assume that $\widetilde{\alpha_{1}} a \in B^{\circ}$ and $\widetilde{\alpha_{1}} a_{\leq r} \in B^{\circ}$. Then for all $r \in \mathbb{N}$, as $n, N \rightarrow \infty$, we have

$$
\mathbb{P}\left\{\eta_{(N)} \leq r\right\}=\left(1-P_{r}\right)^{N}(1+\mathrm{o}(1)) .
$$

\section{PROOFS AND AUXILIARY RESUlTS}

Let $A$ be a fixed event, $\mathbb{P}(A)>0$. Let $\mathbb{P}^{A}$ denote the conditional probability with respect to the event $A$ and let $\mathbb{E}^{A}$ denote the expectation with respect to $\mathbb{P}^{A}$. Let us denote by $\mathbb{I}\{A\}$ the indicator variable of the event $A$. Let us denote by $A_{n N}$ the following event: $A_{n N}=\left\{\sum_{i=1}^{N} \xi_{i} \geq n\right\}$.

Lemma 3 (Model 3.). Assume that (2.1) is satisfied. Let $\frac{4 \sqrt{2} s_{r}}{\sqrt{N}}<\varepsilon<\frac{s_{r}^{3}}{\sqrt{2}}$ be fixed. Then for $N$ large enough, we have

$$
\mathbb{P}\left\{\left|\mu_{n N}-\mathbb{E} \mu_{n N}\right| \geq \varepsilon N\right\} \leq \frac{K}{\mathbb{P}\left(A_{n N}\right)} \frac{\varepsilon^{4} N^{2}}{s_{r}^{4}} e^{-\frac{N \varepsilon^{2}}{32 s_{r}^{2}}},
$$

where $K$ is an absolute constant and $s_{r}^{2}=p_{r}\left(1-p_{r}\right)$. 
Proof. Let $\tau_{i}=\mathbb{I}\left\{\xi_{i}=r\right\}, v=\sum_{i=1}^{N} \tau_{i}, i=1, \ldots, n$. The variance of $\tau_{i}$ is $s_{r}^{2}=$ $p_{r}\left(1-p_{r}\right)$, where $p_{r}=\mathbb{P}\left(\xi_{0}=r\right)$. Let $\tau_{i}^{\prime}$ be an independent copy of $\tau_{i}, i=1, \ldots, N$. Let us denote by $d_{i}$ the variance of $\left(\tau_{i}-\tau_{i}^{\prime}\right)^{2}$. Let $d=\frac{1}{N} \sum_{i=1}^{N} d_{i}$.

In Theorem 2.1 (i) of [4] it was proved that for any fixed event $A$ with $\mathbb{P}(A)>0$ and $\varepsilon^{\prime} \geq 4 \sqrt{2} s_{r}$

$$
\mathbb{P}^{A}\left\{\frac{\left|v-\mathbb{E}^{A} v\right|}{\sqrt{N}} \geq \varepsilon^{\prime}\right\} \leq \frac{\sqrt{2}}{\mathbb{P}(A)} e^{-\frac{\varepsilon^{\prime 2}}{16 s_{r}^{2}}}(1+B),
$$

where

$$
B=B(N, \sigma)=\frac{d}{32}\left(\frac{\varepsilon^{\prime 2}}{8 s_{r}^{4} \sqrt{N}}\right)^{2} f_{2}\left(\frac{2 \varepsilon^{\prime 2}}{8 s_{r}^{4} \sqrt{N}}\right)+\mathrm{O}\left(\frac{8 s_{r}^{2}}{\varepsilon^{\prime 2}}\right)
$$

and

$$
f_{2}(x)=4\left(e^{\frac{x^{2}}{2}}\left(x^{2}+1\right) \Phi(x)+\frac{x}{\sqrt{2 \pi}}\right)-1 .
$$

Here $\Phi(x)$ denotes the cumulative distribution function of the standard normal distribution.

We shall apply Theorem 2.1 (i) of [4] with $A=A_{n N}$ and $\varepsilon^{\prime}=\sqrt{N} \varepsilon$. By (3.2) and (2.1), we obtain

$$
\mathbb{P}\left\{\left|\mu_{n N}-\mathbb{E} \mu_{n N}\right| \geq N \varepsilon\right\}=\mathbb{P}^{A_{n N}}\left\{\left|\nu-\mathbb{E}^{A_{n N}} \nu\right| \geq N \varepsilon\right\} \leq \frac{K}{\mathbb{P}\left(A_{n N}\right)} \frac{\varepsilon^{4} N^{2}}{s_{r}^{4}} e^{-\frac{N \varepsilon^{2}}{32 s_{r}^{2}}},
$$

where $K$ is an absolute constant.

Proof of Theorem 1. It follows from equation (2.3), that either $\xi^{\prime}$ is a (nondegenerate) $p$-stable random variable or $\xi^{\prime}$ is a constant. If $\xi^{\prime}$ is a $p$-stable random variable then its distribution function $F$ is arbitrary many times differentiable (see [9]), therefore it is uniformly continuous. If $\xi^{\prime}$ is a constant, then $F(x)=0, x \leq C+\delta$ for some $0<\delta<\infty$. Therefore, in both cases there exists $0<\delta<\infty$ such that $F(x), x \in(-\infty, C+\delta]$ is a uniformly continuous function and $F(C+\delta)<1$. Let $\frac{n}{N} \in\left(0, a+C \frac{B_{N}}{N}\right]$. Then, using (2.2), we have

$$
\frac{N-1}{B_{N-1}}\left(\frac{n}{N-1}-a-\frac{r}{N-1}\right) \leq C+\delta \text { and } \frac{N}{B_{N}}\left(\frac{n}{N}-a\right) \leq C+\delta
$$

for $n$ and $N$ large enough. Consider the quotient $\frac{\mathbb{P}\left\{\sum_{i=1}^{N-1} \xi_{i} \geq n-r\right\}}{\mathbb{P}\left\{\sum_{i=1}^{N} \xi_{i} \geq n\right\}}$. If $\xi^{\prime}$ is a constant, then, using (3.5),

$$
\frac{\mathbb{P}\left\{\sum_{i=1}^{N-1} \xi_{i} \geq n-r\right\}}{\mathbb{P}\left\{\sum_{i=1}^{N} \xi_{i} \geq n\right\}}=\frac{\mathbb{P}\left\{\frac{1}{B_{N-1}} \sum_{i=1}^{N-1}\left(\xi_{i}-a\right) \geq \frac{n-r-(N-1) a}{B_{N-1}}\right\}}{\mathbb{P}\left\{\frac{1}{B_{N}} \sum_{i=1}^{N}\left(\xi_{i}-a\right) \geq \frac{n-N a}{B_{N}}\right\}}
$$




$$
=\frac{1-\mathbb{P}\left\{\frac{1}{B_{N-1}} \sum_{i=1}^{N-1}\left(\xi_{i}-a\right)<\frac{n-r-(N-1) a}{B_{N-1}}\right\}}{1-\mathbb{P}\left\{\frac{1}{B_{N}} \sum_{i=1}^{N}\left(\xi_{i}-a\right)<\frac{n-N a}{B_{N}}\right\}}=\frac{1-\mathrm{o}(1)}{1-\mathrm{o}(1)} .
$$

Now, consider the case when $\xi^{\prime}$ is a $p$-stable random variable. By (2.3), we have

$$
\begin{aligned}
\frac{\mathbb{P}\left\{\sum_{i=1}^{N-1} \xi_{i} \geq n-r\right\}}{\mathbb{P}\left\{\sum_{i=1}^{N} \xi_{i} \geq n\right\}} & =\frac{1-\mathbb{P}\left\{\frac{1}{B_{N-1}} \sum_{i=1}^{N-1}\left(\xi_{i}-a\right)<\frac{n-r-(N-1) a}{B_{N-1}}\right\}}{1-\mathbb{P}\left\{\frac{1}{B_{N}} \sum_{i=1}^{N}\left(\xi_{i}-a\right)<\frac{n-N a}{B_{N}}\right\}} \\
& =\frac{1-F\left(\frac{N}{B_{N-1}}\left(\frac{n}{N}-a-\frac{r-a}{N}\right)\right)+\mathrm{o}(1)}{1-F\left(\frac{N}{B_{N}}\left(\frac{n}{N}-a\right)\right)+\mathrm{o}(1)} .
\end{aligned}
$$

Therefore we obtain

$$
\begin{aligned}
& \left|1-\frac{\mathbb{P}\left\{\sum_{i=1}^{N-1} \xi_{i} \geq n-r\right\}}{\mathbb{P}\left\{\sum_{i=1}^{N} \xi_{i} \geq n\right\}}\right| \\
& \leq \frac{\left|F\left(\frac{N}{B_{N-1}}\left(\frac{n}{N}-a-\frac{r-a}{N}\right)\right)-F\left(\frac{N}{B_{N}}\left(\frac{n}{N}-a\right)\right)\right|+\mathrm{o}(1)}{1-F(C+\delta)+\mathrm{o}(1)} .
\end{aligned}
$$

Using either (3.6) or (3.7) and the uniform continuity property of $F$, we obtain

$$
\lim _{n, N \rightarrow \infty, 0<\frac{n}{N}<a+C \frac{B_{N}}{N}} \frac{\mathbb{P}\left\{\sum_{i=1}^{N-1} \xi_{i} \geq n-r\right\}}{\mathbb{P}\left\{\sum_{i=1}^{N} \xi_{i} \geq n\right\}}=1 .
$$

Consequently

$$
\begin{aligned}
& \lim _{n, N \rightarrow \infty, 0<\frac{n}{N} \leq a+C \frac{B_{N}}{N}} \frac{1}{N} \mathbb{E} \mu_{n N} \\
& =\lim _{n, N \rightarrow \infty, 0<\frac{n}{N} \leq a+C \frac{B_{N}}{N}} p_{r} \frac{\mathbb{P}\left\{\sum_{i=1}^{N-1} \xi_{i} \geq n-r\right\}}{\mathbb{P}\left\{\sum_{i=1}^{N} \xi_{i} \geq n\right\}}=p_{r} .
\end{aligned}
$$

Let $\varepsilon>0$. By (2.3) and the continuity property of $F$, the above calculation shows that there exists $K_{0}>0, n_{0} \in \mathbb{N}$ such that $\mathbb{P}\left\{A_{n N}\right\}>K_{0}$ for $n, N>n_{0}, 0<\frac{n}{N} \leq$ $a+C \frac{B_{N}}{N}$. Using this inequality and Lemma 3 , we have for $\varepsilon>0$

$$
\begin{aligned}
& \sum_{n, N>n_{0}, 0<\frac{n}{N}<a+C \frac{B_{N}}{N}} \mathbb{P}\left\{\left|\mu_{n N}-\mathbb{E} \mu_{n N}\right| \geq N \varepsilon\right\} \\
\leq & \sum_{n, N>n_{0}, 0<\frac{n}{N}<a+C \frac{B_{N}}{N}} \frac{K}{\mathbb{P}\left(A_{n N}\right)} \frac{\varepsilon^{4} N^{2}}{s_{r}^{4}} e^{\frac{-\varepsilon^{2} N}{32 s_{r}^{2}}}
\end{aligned}
$$




$$
\begin{aligned}
& \leq \sum_{n, N>n_{0}, 0<\frac{n}{N}<a+C \frac{B_{N}}{N}} \frac{K}{K_{0}} \frac{\varepsilon^{4} N^{2}}{s_{r}^{4}} e^{\frac{-\varepsilon^{2} N}{32 s_{r}^{2}}} \\
& =\sum_{n, N>n_{0}, 0<n<a N+C B_{N}} \frac{K}{K_{0}} \frac{\varepsilon^{4} N^{2}}{s_{r}^{4}} e^{\frac{-\varepsilon^{2} N}{32 s_{r}^{2}}} \\
& \leq \sum_{N>n_{0}} \frac{K}{K_{0}}(a+C) N \frac{\varepsilon^{4} N^{2}}{s_{r}^{4}} e^{\frac{-\varepsilon^{2} N}{32 s_{r}^{2}}}<\infty .
\end{aligned}
$$

By the Borel-Cantelli Lemma,

$$
\frac{1}{N} \mu_{n N}-\mathbb{E} \frac{1}{N} \mu_{n N} \rightarrow 0 \quad \text { almost surely }
$$

as $n, N \rightarrow \infty$ such that $0<\frac{n}{N} \leq a+C \frac{B_{N}}{N}$. By (3.9) and (3.10) we see that $\frac{1}{N} \mu_{n N} \rightarrow$ $p_{r}$ almost surely as $n, N \rightarrow \infty$ such that $0<\frac{n}{N} \leq a+C \frac{B_{N}}{N}$. The proof is complete.

Let $\xi_{0}$ satisfy the Cramér condition. The variance of $\xi_{0}$ is $\sigma^{2}$ and $\Phi$ denotes the standard normal distribution function. Then, by Petrov's large deviation theorem (see Theorem 5.23 in [15]) we have

$$
\frac{\mathbb{P}\left\{S_{N}^{c} \geq y \sqrt{N} \sigma\right\}}{1-\Phi(y)}=\exp \left\{\frac{y^{3}}{\sqrt{N}} \lambda\left(\frac{y}{\sqrt{N}}\right)\right\}\left[1+\mathrm{O}\left(\frac{y+1}{\sqrt{N}}\right)\right]
$$

as $y \rightarrow \infty$ and $y=\mathrm{o}(\sqrt{N})$. Here $\lambda(t)=\sum_{k=0}^{\infty} a_{k} t^{k}$ is a power series with coefficients depending on the cumulants of $\xi_{0}-a$ and with radius of convergence $R_{1}>0$.

Lemma 4. Let $\xi_{0}$ satisfy the Cramér condition.

(1) Then we have

$$
\lim _{n, N \rightarrow \infty, \frac{n}{N} \rightarrow a, \sqrt{N}\left(\frac{n}{N}-a\right) \rightarrow \infty} \frac{\mathbb{P}\left\{\sum_{i=1}^{N-1} \xi_{i} \geq n-r\right\}}{\mathbb{P}\left\{\sum_{i=1}^{N} \xi_{i} \geq n\right\}}=1 .
$$

(2) As $n, N \rightarrow \infty$ such that $\frac{n}{N} \rightarrow a$ and $\sqrt{N}\left(\frac{n}{N}-a\right) \rightarrow \infty$, then we have

$$
\begin{aligned}
& \mathbb{P}\left\{\sum_{i=1}^{N} \xi_{i} \geq n\right\} \\
& =\frac{\sigma}{\sqrt{2 \pi N}\left(\frac{n}{N}-a\right)} \exp \left(-N\left(\frac{\left(\frac{n}{N}-a\right)^{2}}{2 \sigma^{2}}-\frac{\left(\frac{n}{N}-a\right)^{3}}{\sigma^{3}} \lambda\left(\frac{\frac{n}{N}-a}{\sigma}\right)\right)\right)(1+\mathrm{o}(1)) .
\end{aligned}
$$


Proof. Let $x=\frac{n}{N}-a>0$. Then we have

$$
\begin{aligned}
& \frac{\mathbb{P}\left\{\sum_{i=1}^{N-1} \xi_{i} \geq n-r\right\}}{\mathbb{P}\left\{\sum_{i=1}^{N} \xi_{i} \geq n\right\}} \\
& =\frac{\mathbb{P}\left\{\frac{1}{\sigma \sqrt{N-1}} \sum_{i=1}^{N-1}\left(\xi_{i}-a\right) \geq \frac{\sqrt{N}}{\sigma \sqrt{N-1}} \sqrt{N}\left(x+\frac{a-r}{N}\right)\right\}}{\mathbb{P}\left\{\frac{1}{\sigma \sqrt{N}} \sum_{i=1}^{N}\left(\xi_{i}-a\right) \geq \frac{1}{\sigma} x \sqrt{N}\right\}}=\frac{A}{B} .
\end{aligned}
$$

Using Petrov's large deviation theorem, we obtain

$$
\begin{aligned}
A= & \left(1-\Phi\left(\frac{\sqrt{N}}{\sigma \sqrt{N-1}} \sqrt{N}\left(x+\frac{a-r}{N}\right)\right)\right) \\
& \times \exp \left\{N\left(\frac{\sqrt{N}}{\sigma \sqrt{N-1}}\left(x+\frac{a-r}{N}\right)\right)^{3} \lambda\left(\frac{\sqrt{N}}{\sigma \sqrt{N-1}}\left(x+\frac{a-r}{N}\right)\right)\right\} \\
& \times\left(1+\mathrm{O}\left(\frac{\frac{\sqrt{N}}{\sigma \sqrt{N-1}} \sqrt{N}\left(x+\frac{a-r}{N}\right)+1}{\sqrt{N}}\right)\right) \\
= & A_{1} A_{2} A_{3}
\end{aligned}
$$

and

$$
\begin{aligned}
B & =\left(1-\Phi\left(\frac{1}{\sigma} x \sqrt{N}\right)\right) \exp \left\{N\left(\frac{1}{\sigma} x\right)^{3} \lambda\left(\frac{1}{\sigma} x\right)\right\} \times\left(1+\mathrm{O}\left(\frac{\frac{1}{\sigma} x \sqrt{N}+1}{\sqrt{N}}\right)\right) \\
& =B_{1} B_{2} B_{3} .
\end{aligned}
$$

Using the approximation

$$
1-\Phi(y)=\frac{1}{\sqrt{2 \pi} y} e^{-\frac{y^{2}}{2}}\left(1+\mathrm{O}\left(\frac{1}{y^{2}}\right)\right) \text { as } y \rightarrow \infty,
$$

we obtain

$$
\begin{aligned}
\frac{A_{1}}{B_{1}}= & \frac{\frac{1}{\sigma} x \sqrt{N}}{\frac{\sqrt{N}}{\sigma \sqrt{N-1}} \sqrt{N}\left(x+\frac{a-r}{N}\right)} \\
& \times \exp \left(\frac{1}{2}\left(\frac{1}{\sigma} x \sqrt{N}\right)^{2}-\frac{1}{2}\left(\frac{\sqrt{N}}{\sigma \sqrt{N-1}} \sqrt{N}\left(x+\frac{a-r}{N}\right)\right)^{2}\right)(1+\mathrm{o}(1)) \\
= & \frac{1}{1-\frac{a-r}{N x}} \exp \left(\frac{1}{2} \frac{1}{\sigma^{2}} N\left(x^{2}-\frac{N}{N-1}\left(x+\frac{a-r}{N}\right)^{2}\right)\right)(1+\mathrm{o}(1))
\end{aligned}
$$




$$
\begin{aligned}
& =\exp \left(\frac{1}{2 \sigma^{2}} N\left(2 x+\frac{a-r}{N}\right)\left(-\frac{a-r}{N}\right)\right)(1-\mathrm{o}(1)) \\
& =1-\mathrm{o}(1)
\end{aligned}
$$

The power series $\lambda(t)$ is convergent, if $n$ and $N$ is large enough, therefore

$$
\begin{aligned}
\ln \left(\frac{A_{2}}{B_{2}}\right)= & N \sum_{k=0}^{\infty} a_{k}\left(\left(\frac{1}{\sigma} \sqrt{\frac{N}{N-1}}\left(x+\frac{a-r}{N}\right)\right)^{k+3}-\left(\frac{1}{\sigma} x\right)^{k+3}\right) \\
= & N \sum_{k=0}^{\infty} a_{k}\left(\frac{1}{\sigma}\left(\sqrt{\frac{N}{N-1}}\left(x+\frac{a-r}{N}\right)-x\right)\right) \\
& \times \sum_{i=0}^{k+2}\left(\frac{1}{\sigma} \sqrt{\frac{N}{N-1}}\left(x+\frac{a-r}{N}\right)\right)^{k+2-i}\left(\frac{1}{\sigma} x\right)^{i} .
\end{aligned}
$$

Therefore

$$
\begin{aligned}
\left|\ln \left(\frac{A_{2}}{B_{2}}\right)\right| \leq & \frac{1}{\sigma}\left|\sqrt{\frac{N}{N-1}}(a-r)+x \frac{\frac{N}{N-1}}{\sqrt{\frac{N}{N-1}}+1}\right| \\
& \times \sum_{k=0}^{\infty}\left|a_{k}\right|\left(\frac{1}{\sigma} \sqrt{\frac{N}{N-1}}\left(|x|+\frac{|a-r|}{N}\right)\right)^{k+2}(k+3) .
\end{aligned}
$$

The radius of convergence of the above series is positive, so we obtain

$$
\left|\ln \left(\frac{A_{2}}{B_{2}}\right)\right|=\mathrm{o}(1) \text {, and } \frac{A_{2}}{B_{2}}=1+\mathrm{o}(1) \text { as } n, N \rightarrow \infty,
$$

because $|x| \rightarrow 0$ and $\frac{|a-r|}{n} \rightarrow 0$.

Finally, it is easy to see that

$$
\frac{A_{3}}{B_{3}} \rightarrow 1
$$

(2) Let $x=\left(\frac{n}{N}-a\right)$. Using (3.15) and approximation (3.16), we obtain

$$
\begin{aligned}
& \mathbb{P}\left\{\sum_{i=1}^{N} \xi_{i} \geq n\right\}=B \\
& =\frac{1}{\sqrt{2 \pi} \frac{1}{\sigma} x \sqrt{N}} e^{-\frac{1}{2}\left(\frac{1}{\sigma} x \sqrt{N}\right)^{2}} \exp \left(N\left(\frac{1}{\sigma} x\right)^{3} \lambda\left(\frac{1}{\sigma} x\right)\right)(1+\mathrm{o}(1)) \\
& =\frac{\sigma}{\sqrt{2 \pi}\left(\frac{n}{N}-a\right) \sqrt{N}}
\end{aligned}
$$




$$
\times \exp \left(-\frac{1}{2 \sigma^{2}} N\left(\frac{n}{N}-a\right)^{2}+N \frac{1}{\sigma^{3}}\left(\frac{n}{N}-a\right)^{3} \lambda\left(\frac{\left(\frac{n}{N}-a\right)}{\sigma}\right)\right)(1+\mathrm{o}(1))
$$

as $n, N \rightarrow \infty, \frac{n}{N} \rightarrow \alpha$ and $\sqrt{N}\left(\frac{n}{N}-a\right) \rightarrow \infty$.

Proof of Corollary 1. We apply Theorem 1 with $B_{N}=N$. We see that, by Kolmogorov's law of large numbers, (2.3) is true with $\xi^{\prime}=0$. So Theorem 1 implies the result.

Proof of Corollary 2. We apply Theorem 1 with $B_{N}=\sqrt{N}$. By the central limit theorem, (2.3) is true if $\xi^{\prime}$ is a Gaussian random variable with expectation 0 and variance $\sigma^{2}$. So Theorem 1 implies the result.

Proof of Theorem 2. (1) (2.5) follows from Corollary 1. have

(2) Let $n, N \rightarrow \infty$ such that $\frac{n}{N} \rightarrow a, \sqrt{N}\left(\frac{n}{N}-a\right) \rightarrow \infty$. By Lemma 4 (1), we

$$
\frac{1}{N} \mathbb{E} \mu_{n N}=p_{r} \frac{\mathbb{P}\left\{\sum_{i=1}^{N-1} \xi_{i} \geq n-r\right\}}{\mathbb{P}\left\{\sum_{i=1}^{N} \xi_{i} \geq n\right\}} \rightarrow p_{r} .
$$

Let $0<\varepsilon<1$. If $n, N \rightarrow \infty$ such that $\frac{n}{N} \rightarrow a, \sqrt{N}\left(\frac{n}{N}-a\right) \rightarrow \infty$, then there exists $n_{0} \in \mathbb{N}$, such that

- $\left|\frac{n}{N}-a\right|<\varepsilon$ for all $n, N>n_{0}$;

- $\sqrt{N}\left(\frac{n}{N}-a\right)>1$ for all $n, N>n_{0}$;

- $\frac{\left(\frac{n}{N}-a\right)^{2}}{2 \sigma^{2}}-\frac{\left(\frac{n}{N}-a\right)^{3}}{\sigma^{3}} \lambda\left(\frac{\frac{n}{N}-a}{\sigma}\right)<\frac{\varepsilon^{2}}{64 s_{r}^{2}}$ for all $n, N>n_{0}$.

As before, $A_{n N}$ denotes the following event: $A_{n N}=\left\{\sum_{i=1}^{N} \xi_{i} \geq n\right\}$. Under the above relations, by Lemma 3 and Lemma 4 (2), for $n, N>n_{0}$ we have

$$
\begin{aligned}
& \sum_{n, N>n_{0}} \mathbb{P}\left\{\left|\frac{1}{N} \mu_{n N}-\mathbb{E} \frac{1}{N} \mu_{n N}\right| \geq \varepsilon\right\} \leq K \sum_{n, N>n_{0}} \frac{1}{\mathbb{P}\left(A_{n N}\right)} \frac{\varepsilon^{4} N^{2}}{s_{r}^{4}} e^{-\frac{N \varepsilon^{2}}{32 s_{r}^{2}}} \\
& \leq K \sum_{n, N>n_{0},\left|\frac{n}{N}-a\right|<\varepsilon} \frac{\sqrt{2 \pi}\left(\frac{n}{N}-a\right) \sqrt{N}}{\sigma \exp \left(-N\left(\frac{\left(\frac{n}{N}-a\right)^{2}}{2 \sigma^{2}}-\frac{\left(\frac{n}{N}-a\right)^{3}}{\sigma^{3}} \lambda\left(\frac{\frac{n}{N}-a}{\sigma}\right)\right)\right)} \frac{\varepsilon^{4} N^{2}}{s_{r}^{4}} e^{\frac{-\varepsilon^{2} N}{32 s_{r}^{2}}} \\
& \leq K_{1} \sum_{n, N>n_{0}, N(a-\varepsilon)<n<(a+\varepsilon) N} \frac{\sqrt{N} \varepsilon}{\sigma} \frac{1}{e^{\frac{-\varepsilon^{2} N}{64 s_{r}^{2}}}} \frac{\varepsilon^{4} N^{2}}{s_{r}^{4}} e^{\frac{-\varepsilon^{2} N}{32 s_{r}^{2}}} \\
& \leq K_{1} \sum_{N=n_{0}}^{\infty} \frac{C \varepsilon^{5}}{\sigma} \frac{\sqrt{N}}{s_{r}^{4}} 2 N^{3} \varepsilon e^{\frac{-\varepsilon^{2} N}{64 s_{r}^{2}}}<\infty
\end{aligned}
$$

Above we used that the number of $n$ 's in the sum in bounded by $2 N C$. Consequently, by the Borel-Cantelli Lemma, $\frac{1}{N} \mu_{n N}-\mathbb{E} \frac{1}{N} \mu_{n N} \rightarrow 0$ almost surely, as $n, N \rightarrow \infty$ 
such that $\frac{n}{N} \rightarrow a, \sqrt{N}\left(\frac{n}{N}-a\right) \rightarrow \infty$. Therefore, by (3.18),

$$
\frac{1}{N} \mu_{n N}=\frac{1}{N} \mu_{n N}-\mathbb{E} \frac{1}{N} \mu_{n N}+\mathbb{E} \frac{1}{N} \mu_{n N} \rightarrow p_{r} \quad \text { almost surely, }
$$

as $n, N \rightarrow \infty$ such that $\frac{n}{N} \rightarrow a, \sqrt{N}\left(\frac{n}{N}-a\right) \rightarrow \infty$. By (3.19), there exists $\Omega_{1} \subset \Omega$ such that $\mathbb{P}\left\{\Omega_{1}\right\}=1$ and for all $\omega \in \Omega_{1}$ we have

$$
\frac{1}{N} \mu_{n N}(\omega) \rightarrow p_{r} \text { as } n, N \rightarrow \infty \text { such that } \frac{n}{N} \rightarrow a, \sqrt{N}\left(\frac{n}{N}-a\right) \rightarrow \infty \text {. }
$$

By Corollary 2, there exists $\Omega_{2} \subset \Omega$ such that $\mathbb{P}\left\{\Omega_{2}\right\}=1$ and for all $\omega \in \Omega_{2}$ we have

$$
\frac{1}{N} \mu_{n N}(\omega) \rightarrow p_{r} \text { as } n, N \rightarrow \infty \text { such that } \frac{n}{N} \rightarrow a, \sqrt{N}\left(\frac{n}{N}-a\right) \leq m
$$

for an arbitrary fixed $m \in \mathbb{N}$.

Let $\omega \in \Omega_{3}=\Omega_{1} \cap \Omega_{2}$. Suppose that $\frac{n}{N} \rightarrow a$, as $n, N \rightarrow \infty$. Let $\varepsilon>0$.

Since $\omega \in \Omega_{1}$, there exist $m=m(\omega), n_{1}=n_{1}(\omega) \in \mathbb{N}$ and $\delta_{1}=\delta_{1}(\omega)>0$ depending on $\omega$ such that $\left|\frac{1}{N} \mu_{n N}(\omega)-p_{r}\right|<\varepsilon$, if $n, N>n_{1}, \sqrt{N}\left(\frac{n}{N}-a\right)>m$ and $\left|\frac{n}{N}-a\right|<\delta_{1}$.

Since $\omega \in \Omega_{2}$, there exist $n_{2}=n_{2}(\omega, m) \in \mathbb{N}$ and $\delta_{2}=\delta_{2}(\omega, m)>0$ depending on $\omega$ and $m$, such that $\left|\frac{1}{N} \mu_{n N}(\omega)-p_{r}\right|<\varepsilon$, if $n, N>n_{2}, \sqrt{N}\left(\frac{n}{N}-a\right) \leq m$ and $\left|\frac{n}{N}-a\right|<\delta_{2}$.

Introduce notation $\delta=\min \left(\delta_{1}, \delta_{2}\right), n_{0}=\max \left(n_{1}, n_{2}\right)$. Consequently, if $n, N>n_{0}$ and $\left|\frac{n}{N}-a\right|<\delta$, then $\left|\frac{1}{N} \mu_{n N}(\omega)-p_{r}\right|<\varepsilon$. The proof is complete.

Proof of Theorem 3. Consider the following represention of the distribution of $\mu_{n N}$ in Model 3 (see Theorem 2.2 of [8]).

For all $k=0,1,2, \ldots, N$,

$$
\mathbb{P}\left\{\mu_{n N}=k\right\}=\left(\begin{array}{l}
N \\
k
\end{array}\right) p_{1}^{k}\left(1-p_{1}\right)^{N-k} \frac{\mathbb{P}\left\{S_{N-k}^{(1)} \geq n-k\right\}}{\mathbb{P}\left\{S_{N} \geq n\right\}} .
$$

Let $k \in \mathbb{N}_{0}$. By the Poisson limit theorem, one has

$$
\left(\begin{array}{l}
N \\
k
\end{array}\right) p_{1}^{k}\left(1-p_{1}\right)^{N-k}=\frac{\lambda^{k} e^{-\lambda}}{k !}(1+\mathrm{o}(1)) .
$$

$N p_{1}(\theta) \rightarrow \lambda$ implies that $\theta \rightarrow 0$, therefore $B(\theta)=b_{0}+\mathrm{o}(1)$ and $\theta=\frac{b_{0} \lambda+\mathrm{o}(1)}{N b_{1}}$. Using Theorem 2 in [11], we have

$$
\mathbb{P}\left\{S_{N} \geq n\right\}=\left(\sum_{l=n}^{\infty} \frac{\lambda^{l} e^{-\lambda}}{l !}\right)(1+\mathrm{o}(1)) .
$$


Since $\mathbb{P}\left\{\xi_{0}^{(1)}=1\right\}=0$, we obtain that

$$
\mathbb{P}\left\{S_{N-k}^{(1)}<n-k\right\} \leq \mathbb{E} S_{N-k}^{(1)} \leq N \mathbb{E} \xi_{0}^{(1)}=N \frac{\frac{b_{2}}{b_{0}}\left(\frac{b_{0} \lambda+\mathrm{o}(1)}{N b_{1}}\right)^{2}}{1-\frac{b_{1}}{b_{0}} \frac{b_{0} \lambda+\mathrm{o}(1)}{N b_{1}}}(1+\mathrm{o}(1))=\mathrm{o}(1) .
$$

Therefore

$$
\left\{S_{N-k}^{(1)} \geq n-k\right\}=1+\mathrm{o}(1) .
$$

Using (3.22), (3.23) and (3.24) in (3.21), we obtain the desired result.

Proof of Lemma 1. The proof is a modification of the proof of Lemma 1.2.1 in [12]. Let us denote by $B-k r=\{x-k r \mid x \in B\}$ for any $B \subset \mathbb{R}_{+}, k, r \in \mathbb{R}_{+}$fixed. Let $A_{k}^{(r)}$ denote the event that exactly $k$ of the random variables $\xi_{1}, \ldots, \xi_{N}$ being equal to $r$. By (2.10), we have

$$
\mathbb{P}\left\{\mu_{n N}=k\right\}=\mathbb{P}\left(A_{k}^{(r)} \mid S_{N} \in B_{n}\right)=\frac{\mathbb{P}\left(A_{k}^{(r)}, S_{N} \in B_{n}\right)}{\mathbb{P}\left(S_{N} \in B_{n}\right)} .
$$

Therefore, using that $\xi_{1}, \ldots, \xi_{N}$ are independent random variables and the event $A_{k}^{(r)}$ can occur $\left(\begin{array}{l}N \\ k\end{array}\right)$ differents ways, we have

$$
\begin{aligned}
\mathbb{P}\left(A_{k}^{(r)}, S_{N} \in B_{n}\right)= & \mathbb{P}\left(S_{N} \in B_{n} \mid A_{k}^{(r)}\right) \mathbb{P}\left(A_{k}^{(r)}\right) \\
= & \left(\begin{array}{l}
N \\
k
\end{array}\right) p_{r}^{k}\left(1-p_{r}\right)^{N-k} \\
& \times \mathbb{P}\left(S_{N} \in B_{n} \mid \xi_{1} \neq r, \ldots, \xi_{N-k} \neq r, \xi_{N-k+1}=r, \ldots, \xi_{N}=r\right) \\
= & \left(\begin{array}{l}
N \\
k
\end{array}\right) p_{r}^{k}\left(1-p_{r}\right)^{N-k} \mathbb{P}\left(S_{N-k}^{(r)} \in B_{n}-k r\right) .
\end{aligned}
$$

Proof of Theorem 4. The proof of our limit theorem is based on representation (2.11). Using the Moivre-Laplace theorem, we have

$$
\left(\begin{array}{l}
N \\
k
\end{array}\right) p_{r}^{k}\left(1-p_{r}\right)^{N-k}=\frac{1}{\sqrt{2 \pi N} s_{r}} e^{-u^{2} / 2}(1+\mathrm{o}(1))
$$

as $N \rightarrow \infty$ and $u=\frac{k-N p_{r}}{s_{r} N^{1 / 2}}$ belongs to a bounded fixed interval, where $s_{r}^{2}=p_{r}(1-$ $\left.p_{r}\right)$.

Let $a<\infty, \widetilde{\alpha_{1}} a \in B^{\circ}$. Apply Kolmogorov's law of large numbers. Then we have

$$
\mathbb{P}\left\{S_{N} \in B_{n}\right\}=\mathbb{P}\left(\frac{N}{f(n)} \frac{S_{N}}{N} \in B\right) \stackrel{n, N \rightarrow \infty}{\longrightarrow} 1,
$$


and

$$
\mathbb{P}\left\{S_{N-k}^{(r)} \in B_{n}-k r\right\}=\mathbb{P}\left(\frac{N-k}{f(n)} \frac{S_{N-k}^{(r)}}{N-k} \in B-\frac{k r}{f(n)}\right) \stackrel{n, N \rightarrow \infty}{\longrightarrow} 1 .
$$

To obtain (3.27), we used that

$$
\frac{N-k}{f(n)}=\frac{N}{f(n)}\left(1-\frac{u s_{r}}{\sqrt{N}}-p_{r}\right) \stackrel{n, N \rightarrow \infty}{\longrightarrow} \widetilde{\alpha_{1}}\left(1-p_{r}\right)
$$

and

$$
\frac{k r}{f(n)}=\frac{N}{f(n)}\left(\frac{u s_{r} r}{\sqrt{N}}+r p_{r}\right) \stackrel{n, N \rightarrow \infty}{\longrightarrow} \widetilde{\alpha_{1}} r p_{r}
$$

Proof of Lemma 2. The proof of Lemma 2 is a modification of the proof of Lemma 1.2.2 in [12].

$$
\begin{aligned}
& \mathbb{P}\left\{\eta_{(N)} \leq r\right\}=\mathbb{P}\left\{\eta_{1} \leq r, \ldots, \eta_{N} \leq r\right\}=\mathbb{P}\left\{\xi_{1} \leq r, \ldots, \xi_{N} \leq r \mid \sum_{i=1}^{N} \xi_{i} \in B_{n}\right\} \\
& =\frac{\mathbb{P}\left\{\xi_{1} \leq r, \ldots, \xi_{N} \leq r, S_{N} \in B_{n}\right\}}{\mathbb{P}\left\{S_{N} \in B_{n}\right\}}=\left(1-P_{r}\right)^{N} \frac{\mathbb{P}\left\{S_{N}^{(\leq r)} \in B_{n}\right\}}{\mathbb{P}\left\{S_{N} \in B_{n}\right\}} .
\end{aligned}
$$

Proof of Theorem 5. Consider representation (2.12) and apply Kolmogorov's law of large numbers for $S_{N}$ and $S^{(\leq r)}$. Then we have

$$
\mathbb{P}\left\{S_{N} \in B_{n}\right\}=\mathbb{P}\left(\frac{N}{f(n)} \frac{S_{N}}{N} \in B\right) \stackrel{n, N \rightarrow \infty}{\longrightarrow} 1,
$$

and

$$
\mathbb{P}\left\{S_{N}^{(\leq r)} \in B_{n}\right\}=\mathbb{P}\left(\frac{N}{f(n)} \frac{S_{N}^{(\leq r)}}{N} \in B\right) \stackrel{n, N \rightarrow \infty}{\longrightarrow} 1 .
$$

\section{REFERENCES}

[1] A. Békéssy, "On classical occupancy problems. I," Publ. Math. Inst. Hung. Acad. Sci., Ser. A, vol. 8, no. 1-2, pp. 59-71, 1963.

[2] A. N. Chuprunov and I. Fazekas, "Inequalities and strong laws of large numbers for random allocations," Acta Math. Hung., vol. 109, no. 1-2, pp. 163-182, 2005, doi: 10.1007/s10474-005-0240-9.

[3] A. N. Chuprunov and I. Fazekas, "Strong laws of large numbers for random forests," Acta Math. Hung., vol. 124, no. 1-2, pp. 59-71, 2009, doi: 10.1007/s10474-009-8150-x.

[4] A. N. Chuprunov and I. Fazekas, "An exponential inequality and strong limit theorems for conditional expectations," Period. Math. Hung., vol. 61, no. 1-2, pp. 103-120, 2010, doi: 10.1007/s10998-010-3103-2. 
[5] A. N. Chuprunov and I. Fazekas, "An inequality for moments and its applications to the generalized allocation scheme," Publ. Math. Debrecen, vol. 76, no. 3, pp. 271-286, 2010.

[6] A. N. Chuprunov and I. Fazekas, "An analogue of the generalised allocation scheme: limit theorems for the maximum cell load," Discrete Math. Appl., vol. 22, no. 3, pp. 307-314, 2012, doi: 10.1515/dma-2012-020.

[7] A. N. Chuprunov and I. Fazekas, "An analogue of the generalised allocation scheme: limit theorems for the number of cells containing a given number of particles," Discrete Math. Appl., vol. 22, no. 1, pp. 101-122, 2012.

[8] I. Fazekas and B. Porvázsnyik, "A generalized allocation scheme,” Ann. Math. Inform., vol. 39, pp. 57-70, 2012.

[9] B. V. Gnedenko and A. N. Kolmogorov, Limit distributions for sums of independent random variables, ser. Addison-Wesley Mathematics Series. Cambridge, Mass.: Addison-Wesley Publishing Company, Inc., 1954.

[10] A. V. Kolchin, "On limit theorems for the generalised allocation scheme," Discrete Math. Appl., vol. 13, no. 6, pp. 627-636, 2003, doi: 10.1515/156939203322733336.

[11] A. V. Kolchin and V. F. Kolchin, "On transition of distributions of sums of independent identically distributed random variables from one lattice to another in the generalised allocation scheme," Discrete Math. Appl., vol. 16, no. 6, pp. 527-540, 2006, doi: 10.1515/156939206779218023.

[12] V. F. Kolchin, Random Graphs, ser. Encyclopedia of Mathematics and Its Applications. Cambridge: Cambridge University Press, 1999, vol. 53.

[13] V. F. Kolchin, B. A. Sevast'yanov, and V. P. Chistyakov, Random allocations. Washington D. C.: V.H. Winston \& Sons, 1978.

[14] Y. L. Pavlov, Random Forests. Utrecht: VSP, 2000.

[15] V. V. Petrov, Limit theorems of probability theory. Sequences of independent random variables, ser. Oxford Studies in Probability. Oxford: Clarendon Press, 1995, vol. 4.

\section{Authors' addresses}

István Fazekas

University of Debrecen, Department of Applied Mathematics and Probability Theory, P.O. Box 12, 4010 Debrecen, Hungary

E-mail address: fazekas.istvaneinf.unideb.hu

Bettina Porvázsnyik

University of Debrecen, Department of Applied Mathematics and Probability Theory, P.O. Box 12, 4010 Debrecen, Hungary

E-mail address: porvazsnyik.bettina@inf . unideb.hu 